\title{
TRADUZINDO O PASSADO: ERINNERN ALS PROZESS DES ÜBERSETZENS AM BEISPIEL VON NOEMI JAFFES O QUE OS CEGOS ESTÃO SONHANDO
}

\author{
Kathrin Sartingen 1 \\ Tatjana Wais ${ }^{1}$ \\ -1Universität Wien, Viena, Áustria
}

\begin{abstract}
Resumo: A presente contribuição coloca o livro $O$ que os cegos estão sonhando de Noemi Jaffe em uma discussão teórica da construção literária da memória. Usando abordagens da teoria literária e da memória, o objetivo é mostrar até que ponto a memória pode ser entendida como um processo de tradução em múltiplas etapas - tanto no sentido tradicional como na tradução entre línguas e culturas quanto no sentido metafórico como tradução de vivência. Este artigo parte do pressuposto de que lembrar é um processo dinâmico e inacabado que se baseia na tradução, recodificação, re-semiotização e realocação das memórias.
\end{abstract}

Palavras-chave: Memória; Tradução; Teoria Literária; Transculturalidade

\section{TRADUZINDO O PASSADO: ERINNERN ALS PROZESS DES ÜBERSETZENS AM BEISPIEL VON NOEMI JAFFES O QUE OS CEGOS ESTÃO SONHANDO}

\begin{abstract}
Der vorliegende Beitrag stellt das Buch $O$ que os cegos estão sonhando in eine gedächtnis- und literaturtheoretische Auseinandersetzung um Erinnerungsdiskurse und Erinnerungsmedien. Ausgehend von der medialen Repräsentierung der Shoah-Erinnerung soll anhand aktueller literaturwissenschaftlicher Ansätze gezeigt werden, inwiefern Erinnerung als ein mehrstufiger Prozess des Übersetzens - sowohl im traditionellen Sinn zwischen den Sprachen und Kulturräumen als auch im symbolischen Sinn als Übersetzung von Erfahrung - verstanden werden kann. Dieser These liegt die Annahme zugrunde, dass Erinnern
\end{abstract}


- auch das an traumatische Ereignisse wie die Shoah - ein dynamischer, unabgeschlossener Prozess ist, der auf dem Übersetzen, Umcodieren, ReSemiotisieren und Verlagern von Gedächtnisinhalten beruht.

Keywords: Erinnerung; Gedächtnis; Übersetzung; Literaturtheorie; Transkulturalität

\title{
TRADUZINDO O PASSADO: REMEMBERING AS A PROCESS OF TRANSLATION ON THE EXAMPLE OF NOEMI JAFFE'S O QUE OS CEGOS ESTÃO SONHANDO
}

\begin{abstract}
The present contribution places the book $O$ que os cegos estão sonhando in a theoretical examination of memory discourses and memory media. Based on the media representation of Shoah memory, current literary approaches will show to what extent memory can be understood as a multi-stage process of translation - both in the traditional sense between languages and cultural areas and in the symbolic sense as a translation of experience. This thesis assumes that remembering - including that of traumatic events such as the Shoah - is a dynamic, unfinished process that is based on the translation, recoding, re-semiotization and relocation of memory contents.
\end{abstract}

Keywords: Memory; Translation; Literary Theory; Transculturality

"Lembrar e esquecer são coisas muito parecidas. São processos mnemônicos seletivos, relacionados ao passado - carregados de invenção e realidade." (Jaffe 165)

\section{Zum Shoah-Gedächtnis: zwischen individueller und kollektiver Erinnerung}

EinesderwohlambestendokumentiertenMenschheitsverbrechen (qtd in Assmann 246), das in den letzten Jahrzehnten in diversen wissenschaftlichen Disziplinen ausgiebig erforscht wurde, ist zweifelsohne die Shoah. Zahlreiche literarische Werke, Dokumentarfilme und Serien, Museen, Gedenkstätten, Dokumentationszentren, Archive, Tagebücher, Monumente, 
Ausstellungen, Theaterstücke und wissenschaftliche Publikationen machen sich den Schatten der europäischen Vergangenheit zum Thema, um dem Vergessen entgegenzuwirken. Die starke mediale und institutionelle Präsenz der Shoah leistet einen wesentlichen Beitrag zur Konstruktion des kulturellen Shoah-Gedächtnisses (Assmann 247) und bringt zugleich die Frage nach der Legitimität der medialen Repräsentation des historischen Ereignisses auf, ein Grundproblem, das die "Erinnerungsgeschichte der Shoah" (Assmann 247) seit jeher begleitet: "como representar algo que vai além da nossa capacidade de imaginar e representar?" (Seligmann-Silva 79)

Die Frage nach der Darstellbarkeit der Shoah zieht sich leitmotivisch durch den Erinnerungsdiskurs der letzten Jahrzehnte: Jegliche - zumal fiktive - Inszenierung jenseits von Authentizität und Faktizität galt lange Zeit als illegitime Grenzüberschreitung, doch hat sich die Perspektive aufgrund der Verschiebung des sozio-kulturellen Bezugsrahmens im Vergleich $\mathrm{zu}$ vorherigen Generationen grundlegend geändert (Assmann 28). Anders als in der Zeit unmittelbar nach dem Zweiten Weltkrieg, in der sich Intellektuelle wie der deutsche Philosoph Theodor Adorno ${ }^{1}$, der deutsche Historiker Dan Diner oder der französische Filmemacher Claude Lanzmann (Shoah, 1985) auf die Shoah als "nachwirkende Präsenz des historischen Ereignisses beziehen”, wird die Shoah heute vor allem über ein "medial gestütztes Gedächtnis" vermittelt (qtd in Assmann 28). In der Gegenwart steht vor allem die Multiperspektivität des Erfahrungsgedächtnisses (qtd in Assmann 49) sowie die Grenzen und Möglichkeiten seiner medialen Inszenierung. Saul Friedländer betont in seinem im Jahr 1993 erschienenen Buch Memory, History and the Extermination of the Jews of Europe, dass die Shoah ein "Grenzereignis" darstelle, das nur in seiner individuellen Erinnerung angemessen dargestellt werden könne. (qtd in Friedländer 1993).

\footnotetext{
${ }^{1}$ In seiner Schrift Kulturkritik und Gesellschaft schreibt Adorno: "nach Auschwitz ein Gedicht zu schreiben, ist barbarisch. " (Adorno 26).
} 
In Zeiten wie diesen, in denen sich - angesichts der aktuellen Corona-Pandemie noch rascher als zuvor - das Erfahrungsgedächtnis der Zeitzeuginnen allmählich auflöst, steht die Erinnerung an die Shoah vor einer Wende: Gespräche mit Überlebenden werden in naher Zukunft nicht mehr möglich sein, was ein Umdenken im Hinblick auf erinnerungskulturelle Praktiken voraussetzt. Der Erinnerungsdiskurs stützt sich zunehmend auf materielle Datenträger und Institutionen, denn "ohne Kodifizierung in Zeichen und Symbolen, ohne Formung in Texten und Bildern gibt es kein kulturelles Gedächtnis" (Assmann 235). Besonders die Künste haben hier eine wichtige Funktion inne, da sie "durch prägnante Formen der Vergegenwärtigung von Vergangenheit" die "historische Imagination" erweitern und eine lebendige Auseinandersetzung mit dem "gespeicherten Wissen" ermöglichen (Assmann 247). Die kulturelle Erinnerung an die Shoah basiert zunehmend weniger auf Zeugenberichten aus erster Hand ("testemunho primário" ${ }^{2}$ ), denn auf Erzählungen und Aufarbeitungen der sogenannten "Generation of Postmemory" (Hirsch 29), der zweiten und dritten Generation der Nachgeborenen ("testemunho secundário"; Seligmann-Silva 126), die ihre Familiengeschichte literarisch aufarbeiten und die - im kommunikativen Gedächtnis verankerten - (traumatischen) Erinnerungen ihrer Vorfahren "übersetzen" 3 und "re-semiotisieren" (Assmann 124).

Zahlreiche zeitgenössische brasilianische AutorInnen wie Noemi Jaffe (O que os cegos estão sonhando 2012), Michel Laub (Diário da Queda, 2012), Heliete Vaitsman (O cisne e o aviador 2014), Luize Valente (Sonata em Auschwitz, 2017) und Giselda Leirner (Nas águas do mesmo rio, 2005) - um nur einige zu

\footnotetext{
${ }^{2}$ Seligmann-Silva differenziert hier zwischen "testemunho primário" (HolocaustÜberlebende thematisieren ihre persönliche Erfahrung aus "erster Hand") und "testemunho secundário" (Nachgeborene), (Seligmann-Silva 126).

${ }^{3}$ Nach Aleida Assmann ist Erinnern als ein Prozess des Übersetzens zu verstehen: "Ja, wir können geradezu sagen: erinnern ist übersetzen, und damit bleiben Erinnerungen zugleich in plastischer Bewegung". (Assmann, Der lange Schatten der Vergangenheit. Erinnerungskultur und Geschichtspolitik, 124).
} 
nennen - greifen in ihren Texten die Shoah als individuelles bzw. kollektives Schicksal auf und tragen so zur Vergegenwärtigung des historischen Ereignisses als Teil der brasilianischen (und europäischen) Kulturgeschichte bei.

Der vorliegende Beitrag zeigt am Beispiel von $O$ que os cegos estão sonhando von Noemi Jaffe auf, inwiefern die literarische Erinnerungskonstruktion als ein mehrstufiger Prozess des Übersetzens - sowohl im traditionellen Sinn als Übersetzen zwischen den Sprachen und Kulturräumen, als aber auch im metaphorischen Sinn als Übersetzen von Erfahrung - gedeutet werden kann. Angelehnt an die Transtextualitätstheorie von Gérard Genette (1997) sowie an die gedächtnisorientierte Intertextualitätstheorie von Renate Lachmann (1990) wird der Frage nachgegangen, inwiefern trans- bzw. hypertextuelle Beziehungen im Werk $O$ que os cegos estão sonhando eine erinnerungskulturelle Funktion innehaben und welche Rolle diese im Hinblick auf die mediale Konstruktion des kulturellen Shoah-Gedächtnisses spielen. Dabei soll nicht nur der unmittelbare Zusammenhang zwischen Literatur, Übersetzen und Erinnern beleuchtet, sondern eine neue Perspektive auf die zeitgenössische brasilianische Erinnerungsliteratur und deren erinnerungskulturelle Relevanz bezüglich der Aufarbeitung der nationalsozialistischen Vergangenheit Europas dargelegt werden.

\section{O que os cegos estão sonhando als Literatur zweiter Stufe}

"Das Gedächtnis des Textes ist seine Intertextualität" (Lachmann 35) schreibt die deutsche Literaturtheoretikerin Renate Lachmann in ihrem Buch Gedächtnis und Literatur. Intertextualität in der russischen Moderne (1990). Literaturwissenschaftliche Konzepte von Gedächtnis unterscheiden zwischen der poststrukturalistischen Vorstellung eines Gedächtnisses der Literatur (Erll, Kollektives Gedächtnis und Erinnerungskulturen. Eine Einführung, 62) als Symbol und Sozialsystem und Gedächtnis in der Literatur als hermeneutisches Konzept. Während das 
Gedächtnis der Literatur im Sinne eines genitivus subjectivus auf die intertextuelle Anlage literarischer Texte sowie auf Kanonbildung und Literaturgeschichtsschreibung verweist, beschreibt das Konzept Gedächtnis in der Literatur Aspekte der literarischen Inszenierung von Erinnerung. Ersteres basiert auf der Vorstellung, dass literarische Texte nur in ihrem "diachronen Zusammenhang" verstanden werden können: Durch intertextuelle bzw. transtextuelle Verflechtungen erinnert Literatur nicht nur an sich selbst, sondern ermöglicht durch spezifische Textbezüge neue Bedeutungskonstruktionen, ein "Wieder-Aufladen von Elementen überlieferter Texte mit Bedeutung" (Erll, Kollektives Gedächtnis und Erinnerungskulturen. Eine Einführung, 62).

Die vielfältigen Beziehungen zwischen literarischen Texten subsumiert der französische Literaturtheoretiker Gérard Genette unter den Begriff der "Transtextualität" und beschreibt damit die transtextuelle Beschaffenheit literarischer Texte. Genette fasst in seiner Theorie den Intertextualitätsbegriff enger, indem er zwischen folgenden Formen literarischer Transzendenz differenziert: Intertextualität, Paratextualität, Metatextualität, Architextualität und Hypertextualität (Genette 5). Dieser These zufolge kann ein literarisches Werk als "Literatur auf zweiter Stufe" (Genette 5) verstanden werden, das ohne den Bezug zu anderen Texten bzw. Architexten in dieser Form nicht existieren würde. Die literarische Transzendenz eines Textes, die sich im Sinne Genettes in seiner Beziehung zu einem oder mehreren Texten manifestiert, ist essenziell im Hinblick auf die literarische Konstruktion von Erinnerung: durch transtextuelle Kontakte erinnert sich Literatur nicht nur an andere literarische Texte, sondern vereint auf diese Weise - insbesondere im Hinblick auf die Aufarbeitung der Shoah - unterschiedliche Erinnerungsdiskurse, die miteinander in Dialog treten und so die Pluralität des Erfahrungsgedächtnisses inszenieren.

Diese Pluralität wird in $O$ que os cegos estão sonhando besonders deutlich. So ist das Buch von Noemi Jaffe, das auf mehreren Stufen der Übersetzung und Re-semiotisierung basiert, 
als Hypertext des von ihrer Mutter Lili Jaffe verfassten Tagebuchs (Hypotext) zu verstehen. Indem es von den Bezügen zwischen verschiedenen Texten - genauer: verschiedenen Formen von Tagebüchern - lebt, erwirkt es eine Verwebung des Gesagten/ Geschriebenen, das erst in diesem Zusammenspiel seine ureigene Vielfalt erschafft und zu einem literarischen Geflecht auf zweiter Stufe wird. $O$ que os cegos estão sonhando als Text auf zweiter Stufe bildet damit den gesamten vielschichtigen Prozess von Erinnerungen und Gedächtnisleistungen nach. Dieser mehrstufige Prozess des Übersetzens, Transformierens und Verlagerns, der dem ausgewählten Primärtext zugrunde liegt, soll im Folgenden näher erläutert werden.

\section{Übersetzung auf verschiedenen Stufen: Transposition des serbischen Tagebuchs ins Portugiesische}

The most visible form of transposition is transposing a text from one language to another. (Genette 214)

Das Buch $O$ que os cegos estão sonhando (2012) setzt sich aus drei Teilen zusammen: 1 . dem a posteriori und ursprünglich auf Serbisch verfassten Tagebuch der Shoah-Überlebenden Lili Jaffe " $O$ diário de Lili Jaffe", das anschließend ins Portugiesische übersetzt wurde, 2. dem von der Tochter Noemi Jaffe geschriebenen und aus mehreren kurzen Kapiteln bestehenden literarischen Werk $O$ que os cegos estão sonhando und 3. dem letzten Teil Aqui, lá von Leda Cartum, der Enkelin von Lili Jaffe und Tochter Noemi Jaffes. Das Werk vereint somit die Perspektiven dreier Frauen und dreier Generationen, die die traumatische Erfahrung der Shoah als Teil ihres individuellen Lebens bzw. ihrer Familiengeschichte aufarbeiten und zentrale Fragestellungen der Zeugenschaft und des Spannungsverhältnisses von Erinnern und Vergessen - vor dem Hintergrund des sich stetig auflösenden, kommunikativen ShoahGedächtnisses - aufgreifen. 
Ausgangspunkt des von Noemi Jaffe verfassten postmemorialen Erinnerungsdiskurses in $O$ que os cegos estão sonhando bilden also die traumatischen Erfahrungen der Mutter Lili Jaffe, die im Zweiten Weltkrieg im Alter von 17 Jahren von den Nationalsozialisten festgenommen und anschließend in den Konzentrationslagern Auschwitz und Bergen-Belsen gefangen gehalten wurde. Im Jahr 1945, unmittelbar nach der Befreiung durch das Rote Kreuz in Malmö, hält Lili Jaffe ihre Erinnerungen an die Geschehnisse während und nach dem Zweiten Weltkrieg a posteriori in Tagebuchform fest. Es handelt sich also nicht um ein Tagebuch per se, sondern um eine narrative Konstruktion von Erinnerungen aus der Retrospektive, die die Diskrepanz von "Eindruck" und "Ausdruck" (Assmann 124), zwischen Erinnertem und Erlebtem, ins Zentrum rückt. Die Tatsache, dass das Tagebuch im Nachhinein verfasst wurde, ändert nicht die Direktheit und Unmittelbarkeit der darin niedergeschriebenen Erinnerungen, doch finden im Zuge seiner Rezeption mögliche Verlagerungen, Verschiebungen oder Verdrängungen der traumatischen Gedächtnisinhalte statt. Traumatische Erinnerungen sind - so Arthur Nestrovski und Márcio Seligmann-Silva - grundsätzlich von "Verschiebung" und "Unvollständigkeit" geprägt: "A característica essencial do trauma é o adiamento, ou incompletude do que se sabe" (Nestrovski und Seligmann-Silva 8).

Die "Unvollständigkeit" bzw. "Unzuverlässigkeit” der Erinnerung ${ }^{4}$ (qtd in Assmann, Einführung in die Kulturwissenschaft . Grundbegriffe, Themen, Fragestellungen, 184) spiegelt sich auch in der Struktur des Tagebuchs wider: die Erinnerungsfragmente sind zwar großteils mit genauen Zeit- und Ortsangaben versehen, doch einige Tagebucheinträge sind mit Fragezeichen gekennzeichnet und auch die zeitliche Abfolge der Einträge ist nicht durchwegs chronologisch, was paradigmatisch für den fragmentarischen

4 Aleida Assmann spricht von der "Unzuverlässigkeit von Erinnerungen" (Assmann, Einführung in die Kulturwissenschaft. Grundbegriffe, Themen, Fragestellungen, 184).

Cad. Trad., Florianópolis, v. 41, no 3 p. 132-155, set-dez, 2021. 
Charakter des Erinnerns steht. Lili Jaffe bedient sich zudem bei der Schilderung der Geschehnisse unterschiedlicher Zeitformen, nicht als bewusst gewähltes literarisches Darstellungsverfahren oder stilistisches Mittel, sondern als Kennzeichen ihres individuellen Sprachstils:

Ao conversarem, a filha vê que ela mistura os tempos verbais em português, usa alguns inadequadamente e fala a língua muito mais por hábito e intuição do que por alguma consciência linguística. Durante o processo de tradução do diário, ela também teve uma dificuldade muito grande em traduzir de forma apropriada os tempos verbais. (Jaffe 191)

Auch im serbischen Originaltagebuch der Mutter finden sich diese idiolektalen Verschiebungen, insbesondere bei den Zeitformen. Die Entscheidung, den Inhalt sowie die sprachlichen Partikularitäten des serbischen Originals in der portugiesischen Übersetzung möglichst unverändert beizubehalten, trägt dazu bei, die Authentizität des Ursprungstextes zu wahren und die darin enthaltenen Erinnerungen lebendig zu gestalten. Die portugiesische Übersetzung des Tagebuchs zeichnet sich vor allem durch diese sprachlichen und formalen Besonderheiten aus, die die "Spontanität und Intensität [...], in der es geschrieben wurde" ${ }^{5}$ wahren soll: "Escolhi preservar essas pequenas confusões, justamente para ser fiel ao que a minha mãe sentia no momento em que tomou a decisão de escrever." (Jaffe 8) Die linguistische Transposition ("Translation"; Genette 214) vom Serbischen ins Portugiesische bildet somit nach Genette die erste Stufe des mehrstufigen Erinnerungsprozesses. Dieses Konzept schließt die eigentlich erste Übersetzung, nämlich die Übersetzung

5 “Decidi manter aspectos particulares da escrita da minha mãe no diário, para preservar a espontaneidade e a intensidade com que ele foi escrito, daí o uso peculiar, muitas vezes das concordâncias de gênero e número." (Jaffe 7-8; Übersetzung ins Deutsche und Hervorhebung durch die Verf.). 6 .

Cad. Trad., Florianópolis, v. 41, no 3 p. 132-155, set-dez, 2021. 
der individuellen traumatischen, körperlichen Erfahrung in Schrift, nicht mit ein. An dieser Stelle ist zu überlegen, inwiefern Erinnerungskonzepte dazu beitragen könnten, die narratologischen Theorien zu erweitern, indem körperliche, non-verbale, mündlich vorhandene Texte ebenfalls als Hypotexte, als konzeptuelle Stufen anzusehen sind, die in schriftliche Realisierung, d.h. in Schriftsprache übersetzt werden.

Das Tagebuch, dessen serbisches Original sich heute in der internationalen Shoah-Gedenkstätte Yad Vashem in Jerusalem befindet, fungiert nämlich zusammen mit den mündlichen Aufzeichnungen als Hypotext, dessen Inhalt und Form möglichst nah am Ausgangstext entlang in den portugiesischsprachigen Hypertext transformiert wurden. Die Übersetzung der Tagebuchaufzeichnungen ins Portugiesische sowie ihre "Resemiotisierung" im Erinnerungsprojekt Noemi Jaffes machen das Tagebuch $\mathrm{zu}$ einem bedeutenden Text der brasilianischen Zeugenliteratur.

\section{O que os cegos estão sonhando als Hypertext des Tagebuchs}

Erinnern bedeutet immer weniger, sich auf eine Geschichte zu besinnen, und immer mehr, ein Bild aufrufen zu können.

(Sontag 104)

Die nächste Stufe des Übersetzens im Erinnerungsprozess bildet die Re-Semiotisierung der im serbischen bzw. portugiesischen Tagebuch festgehaltenen Erinnerungen aus der Perspektive der Nachgeborenen. Das Tagebuch von Lili Jaffe fungiert als Hypotext des später verfassten Hypertextes $O$ que os cegos estão sonhando. Ausgangspunkt für die im Werk dargelegten post-memorialen Reflexionen der Tochter bildet die Lektüre und literarische Bearbeitung ausgewählter Textausschnitte aus dem Tagebuch bzw. Auszüge aus Gesprächen mit der Mutter. (Jaffe 97) Wie ein roter Faden ziehen sich die Erinnerungsfragmente durch den literarischen Text und prägen so die Struktur des Werkes, die sich durch eine 
Aneinanderreihung kurzer essayartiger Texte auszeichnet. Durch transtextuelle Verflechtungen tritt $O$ que os cegos estão sonhando in Dialog mit dem Tagebuch der Mutter und ermöglicht so einen Perspektivwechsel, der sich unter anderem in der Wahl der Erzählperspektive zeigt. Während Lili Jaffe ihre Erinnerungen aus der Perspektive einer Ich-Erzählerin schildert, bedient sich Noemi Jaffe in $O$ que os cegos estão sonhando einer Erzählinstanz in der dritten Person, die bewusst in den Hintergrund tritt und sich von den traumatischen Erfahrungen der Mutter distanziert, um der Rolle der "Stimmträgerin" gerecht zu werden. Als Tochter und somit nicht unmittelbar Betroffene problematisiert diese Erzählinstanz ihre Funktion als "porta-voz":

\begin{abstract}
A filha [...] não se sente bem contando esta história-nãohistória em primeira pessoa. Não foi com ela que as coisas aconteceram. Ela é uma voz e só quer ser voz. [...] A terceira pessoa, como o doutor Pasavento do romance Vila-Matas, quer desaparecer. Aqui, nesta história, é claro que ela não alcança este poder supremo e inestimável do desaparecimento, mas se aproxima mais dele do que se fosse a primeira pessoa. ${ }^{7}$ (Jaffe 188)
\end{abstract}

Damit wirft der narrative Erinnerungsdiskurs im Text nicht nur Licht auf die individuelle Lebenserfahrung Lili Jaffes sowie auf deren Weiterleben als Shoah-Überlebende in der jüdischen Diaspora in Brasilien, sondern greift zentrale Fragestellungen auf, die die "Generation of Postmemory" (qtd in Hirsch 29) beschäftigen. Auf Wunsch der Mutter fungiert die Tochter als Sprachrohr und gedenkt jener Lebensepisoden, die von der Mutter andernfalls verdrängt würden:

${ }^{7}$ Nicht umsonst heißt das Kapitel "Terceira-pessoa”, (Jaffe 188).

Cad. Trad., Florianópolis, v. 41, no 3 p. 132-155, set-dez, 2021. 
Quando o dono da voz esqueceu suas palavras, ou sequer chegou a formulá-las e aparece alguém querendo portar sua voz, ele deixa. Lembre por mim as coisas que eu esqueci. Mas não me conte. Não quero lembrar. Isso você faz só por você; não por mim. Te dou minha voz, minha memória, porque não me importo com elas. Você é que se importa. Então vá e faça. Fique com elas e bom proveito. (Jaffe 163-164)

Hier wird besonders die Ambivalenz von Erinnern und Vergessen im Kontext der Vergangenheitsbewältigung der Shoah deutlich: Während sich die Betroffene Lili Jaffe nicht weiterhin mit ihrer traumatischen Vergangenheit beschäftigen möchte und kann, sieht es die Tochter als ihre Aufgabe, den schrecklichen Ereignissen im Zweiten Weltkrieg - insbesondere vor dem Hintergrund der schwindenden Zeitzeuginnen - zu gedenken und die individuelle Überlebensgeschichte ihrer Mutter einer breiteren Öffentlichkeit zugänglich zu machen. Der transtextuelle Kontakt zwischen dem Mutter-Tagebuch und dem Tochter-Text $O$ que os cegos estão sonhando wirft nicht nur Licht auf die Vergangenheit der Mutter, sondern setzt diese in Bezug zur narrativen "Gegenwart", zum Leben der Shoah-Überlebenden in der Diaspora und ihrer Beziehung zur Tochter.

Im Kapitel "Pedra" thematisiert die Erzählinstanz ein besonders traumatisches Ereignis, das der Mutter während ihres Lageraufenthalts in Auschwitz widerfuhr: "O oficial disse que ia me matar, mas eu pedi perdão e então ele resolveu só me castigar. Fiquei um dia inteiro de joelhos, parada, sobre cascalhos, sustentando uma pedra enorme sobre a cabeça." (Jaffe 112) Der "Stein" ("a pedra") als Figur steht sinnbildlich für die transgenerationelle Übertragung von Traumata im Generationengedächtnis:

Não há como dramatizar ou metaforizar esta pedra. E mesmo assim, ela é o acontecimento, o fato que mais está presente na memória, dela e das filhas. [...] Saber que ela 
sustentou uma pedra durante um dia inteiro, que machucou para sempre o joelho, e tudo isso porque sua prima, e não ela, tinha roubado um pedaço de manteiga, provoca, na memória de quem ouve esta história, na memória de suas filhas, um empuxe gravitacional permanente, [...]. E, no entanto, não se deve sobrevalorizar, transformar a pedra em ícone, em motivo de sofrimento e choque, se ela mesma não permite que seja assim. Que direito têm os outros de sofrer mais do que ela mesma? (Jaffe 112-113)

Durch sogenannte "flashes of imagery" werden individuelle Erinnerungensystematischinnerhalbderselbenoderaufnachfolgende Generationen übertragen, ein Phänomen das Marianne Hirsch als "memorial transmission" bezeichnet (Hirsch 32). Eine solche Funktion übt der Stein bei Noemi Jaffe aus: Er dient als Überträger oder Bild-Träger von Erinnerungen, er nimmt metaphorische Ausmaße an und vervielfältigt Bedeutungszuschreibungen, letztlich dient er in jedem Fall als bildhafte Brücke zur Bewahrung von Erinnerungen.

Anders als das institutionalisierte, archivierende kulturelle Gedächtnis, das auf der Überlieferung von Symbolsystemen basiert, löst sich das auf Kommunikation gestützte soziale Gedächtnis mit dem Schwinden der Zeitzeuginnen allmählich auf. Durch die mündliche und schriftliche Interaktion zwischen Mutter und Tochter wird ein Teil des sozialen Familiengedächtnisses literarisch rekonstruiert und festgehalten. Die Tochter fungiert hier als Zuhörerin und Zeugin, indem sie die Erinnerungen ihrer Mutter ins kulturelle Gedächtnis überführt. Jeanne Marie Gagnebin betont in ihrem Text Memória, História, Testemunho die Wichtigkeit eines erweiterten Konzepts der Zeugenschaft, das einen Dritten einschließt, der nicht dem "höllischen Kreis von Folterer und Gefoltertem angehört" ${ }^{8}$, der zum Sprachrohr wird und in seinen

${ }^{8}$ Gagnebin bezieht sich hierbei auf Hélène Piralian und Janine Altounian: “ [...] muito mais em restabelecer o espaço simbólico onde se possa articular aquele

Cad. Trad., Florianópolis, v. 41, no 3 p. 132-155, set-dez, 2021. 
Worten die unerträgliche Geschichte des Anderen weitergibt: “ [...] Testemunha [...] seria aquele que não vai embora, que consegue ouvir a narração insuportável do outro e que aceita que suas palavras levem adiante, como num revezamento, a história do outro." (Gagnebin 57)

Die heterodiegetische Erzählinstanz in Jaffes $O$ que os cegos estão sonhando thematisiert die Bedeutung individuellen Erinnerns für die Konstruktion eines kollektiven bzw. kulturellen ShoahGedächtnisses. Dieses sei letztlich die Summe aller persönlichen Erfahrungen, die das kollektive Gedächtnis stütze und Bewusstsein für die Vergangenheit einer Gesellschaft schaffe:

\begin{abstract}
É possível que o holocausto já tenha se esgotado e cansado enquanto fonte de aprendizado. Mas isso, supostamente, em seu sentido coletivo. Como experiência individual, não existe tal coisa como cansaço do que foi vivido. O passado individual não se esgota, nem deve se esgotar. E acontece que, na realidade, a vivência coletiva da guerra é uma coleção de memórias individuais; [...] E mesmo assim, que sentido faz contar mais uma história de sofrimento individual para o público? [...] As motivações são obscuras e a filha e a mãe sentem que elas precisam ser expostas: para que elas aprendam alguma coisa, para que a micro história da mãe receba um lugar inscrito no corpo do mundo. [...] (Jaffe 184-185)
\end{abstract}

Nach Assmann liegt die Gefahr im Hinblick auf die Zukunft der Shoah-Erinnerung nicht im Vergessen, sondern in der "Verflachung und Verengung der Erinnerung" (Assmann 246),

que Hélène Piralian e Janine Altounian chamam de 'terceiro' - isto é, aquele que não faz parte do círculo infernal do torturador e do torturado, do assassino e do assassinado [...]." (Gagnebin 57; Übersetzung ins Deutsche und Hervorhebung durch die Verf.) 
ein Thema, das auch die Erzählinstanz in $O$ que os cegos estão sonhando zur Sprache bringt. Das Gedächtnis der Mutter wird im Werk als "memória fixa" beschrieben, da es in erster Linie die im Tagebuch niedergeschriebenen Erinnerungen sind, die auch in den zahlreichen Gesprächen mit der Mutter immer wiederkehren: "A memória dela é uma caixa preta que caiu no mar.” (Jaffe 197) Es scheint, als wären es jene Erinnerungen, die Lili Jaffe unmittelbar nach ihrer Befreiung zu Papier bringt, die vor dem Vergessen bewahrt sind, was nicht zuletzt auf das Verhältnis von Schreiben und Erinnern hinweist. Durch die transtextuelle Verwobenheit der beiden Texte wird ein Erinnerungskonstrukt geschaffen, das die Erinnerungen der Mutter revitalisiert und einen neuen Zugang zur Vergangenheit ermöglicht.

\section{$O$ que os cegos estão sonhando als "Gedächtnis der Vielstimmigkeit"}

Neben den transtextuellen Beziehungen zwischen $O$ que os cegos estão sonhando (Hypertext) und dem Tagebuch (Hypotext) zeichnet sich das Werk durch weitere Textkontakte aus, die für den Erinnerungsdiskurs im Werk von Bedeutung sind. In den Kapiteln "Amor" und "Amor 2" rekonstruiert die Erzählinstanz eine Liebesgeschichte der Mutter, die aufgrund des Lageraufenthalts zu Ende ging: "Antes eu tinha um namorado, e no campo, quando os meninos marchavam, eu ficava procurando por ele" (Jaffe 145). Die Tochter versucht, die Erzählungen ihrer Mutter nachzuvollziehen, was in einen Gedankenstrom der Tochter über die Möglichkeiten der Liebe unter prekären Umständen mündet:

Namorar no campo de concentração. São muito poucos, talvez nenhum, os relatos de namoro nestas condições. Mas são muitos os depoimentos sobre distração e até algum prazer. Primo Levi e Ruth Klüger contam que quando recitavam poemas e trechos de romances, esqueciam que 
estavam no campo e que os outros, ao ouvi-los, conseguiam ter verdadeiros momentos de alegria. (Jaffe 145)

Durch Verweise auf andere literarische Texte wie beispielsweise Fernando Pessoas Fausto. A tragédia subjectiva, der selbst eine "Transformation" bzw. einen "Hypertext" (Genette 7) von Goethes Faust darstellt und das Kapitel "Amor 2" einleitet, werden grundlegende, existentialistische Fragen wie die nach der Liebe aufgegriffen:

Amo como ama o amor. Não conheço nenhuma outra razão para amar senão amar. Que queres que te diga, além de que te amo, se o que quero dizer-te é que te amo? O amor é grande e cabe nesta janela sobre o mar. (Fausto. A tragédia subjectiva, zit. nach: Jaffe 149)

Diese Verweise stellen Möglichkeiten zur Beantwortung der texteigenen Fragen dar, u.a. auf die Frage nach der Liebe, die die Tochter in ihrer Auseinandersetzung mit dem Schicksal der Mutter beschäftigt: "Por favor, expliquem o que é o amor. Expliquem, porque, no campo de concentração, procura-se pelo namorado. $\mathrm{O}$ amor é um consolo? Uma necessidade? Uma benção? É o sentido da vida?" (Jaffe 151)

Durch die Bezugnahme auf den Fauststoff wird die Frage nach der Liebe und ihrer Bedeutung, die den Menschen seit seiner Existenz begleitet, aufgegriffen und vor dem Hintergrund der Shoah problematisiert.

Weitere transtextuelle Bezüge zu Shoah-Überlebenden wie Elie Wiesel, Jacques Le Goff und Jorge Semprún werfen unter anderem ein Licht auf Divergenzen im Erinnerungsdiskurs innerhalb der jüdischen Schicksalsgemeinschaft. Der "kategorische Imperativ" (Gagnebin 100), sich zu erinnern und die Shoah als kollektives jüdisches Schicksal niemals $\mathrm{zu}$ vergessen, tritt hier in ein Spannungsverhältnis mit der Perspektive der Mutter, die ihre Wut 
zugunsten ihrer eigenen Zuversicht unterdrückt. "Eu não tenho raiva de ninguém. Nem dos nazistas. Não gosto de ter raiva" (Jaffe 116). Diese Haltung der Mutter, die - zum Unverständnis ihrer Tochter - trotz der traumatischen Erfahrungen im Konzentrationslager keine Wut den Nationalsozialisten gegenüber empfindet, steht im Gegensatz zur Position anderer Zeitzeuginnen wie Elie Wiesel, Jacques Le Goff, Ruth Klüger, Primo Levi und Jorge Semprún und veranschaulicht die "Multiperspektivität des Erfahrungsgedächtnisses” (Assmann 247):

As filhas sentem raiva por ela não sentir raiva? Primo Levi tem raiva, Ruth Klüger tem raiva, Jorge Semprún tem raiva. Para lembrar é preciso ter raiva; para ter paz é preciso esquecer. $\square$ Não esquecerei jamais esta noite, a primeira noite de acampamento que fez de minha vida uma longa noite sete vezes amaldiçoada. Não esquecerei jamais as chamas que consumiram para sempre a minha féc é o juramento de Elie Wiesel. Jacques Le Goff diz que \ser judeu é recordarロ. Como manter fé e recordação? (Jaffe 117)

Es wird deutlich, dass es für die Erzählinstanz schwierig ist, die Gefühle der Mutter angesichts ihrer Leidensgeschichte nachzuvollziehen und dennoch wird die Unterdrückung von Wutgefühlen auch als eine Charaktereigenschaft der Nachgeborenen beschrieben: "Todas as filhas têm dificuldade, como ela, de sentir raiva" (Jaffe 116). Die Tochter sieht in der Unterdrückung von Wut den "chirurgischen Prozess des Vergessens", denn Wut zu empfinden bedeutet: "alimentar a memória de fatos e histórias para que elas (a memória e a raiva) possam manterse continuamente" (Jaffe 116).

\section{A língua alemã como língua da ordem e do dizível}

Im Kapitel "Raiva" reflektiert die Erzählinstanz nicht nur über das Thema Wut, sondern ebenfalls über ihr Verhältnis zur 
deutschen Sprache, die im Kapitel als "Língua do dizível” und "Língua da ordem" bezeichnet wird. "Língua da ordem" bezieht sich hier in erster Linie auf den Gebrauch der deutschen Sprache im Konzentrationslager: "Em contraposição á língua da literatura, não existe língua mais ordenada do que a do campo de concentração. Lá, a língua chegou ao nível mais apurado, organizado, o nível mais eficiente de comunicação" (Jaffe 118). Zahlreiche deutsche Worte wie "schnell", "nicht", "trinkt", "essen", "Geld", die in den portugiesischen Hypertext integriert wurden, prägen die Erinnerungen der Mutter an den Lageraufenthalt:

Schnell, uma palavra que ocupou a memória de sua adolescência, aterrorizando seus sonhos e remetendo aos soldados que comandavam a mãe e que sempre lhe diziam: schnell, schnell, impondo um ritmo impossível aos prisioneiros, acontecia viva na Alemanha, onde se ouve schnell em todo lugar e a qualquer momento. (Jaffe 118-119)

Die deutsche Sprache als "Língua da ordem" wird unmittelbar mit der deutschen Kultur und der peniblen Ordnung und Organisation der nationalsozialistischen Verbrechen während des Zweiten Weltkriegs in Verbindung gebracht. Einerseits war es eben diese Ordnung, die es den Deutschen ermöglichte, ihre Gräuel in die Tat umzusetzen, andererseits war es vor allem diese Ordnung, die dazu führte, dass diese ihre Verbrechen haargenau dokumentierten und sich letzten Endes selbst verrieten.

O que se vê no campo, por toda parte, além das sombras da morte, é organização, outra forma, ou, na verdade, a linguagem da morte. Talvez seja por isso, mesmo que indiretamente, que tanto ela quanto todas as filhas primem por uma desorganização que beira o infantilismo. [...] Como se isso fosse uma paga ao sofrimento dela, uma vingança à organização alemã. (Jaffe 121) 
Hier wird besonders die Ablehnung der Mutter und deren Töchter im Hinblick auf Organisation und Ordnung deutlich: die Erzählinstanz positioniert sich in klarer Abgrenzung zur Vergangenheit der Mutter, was sich in einem regelrechten "Stolz auf Unordentlichkeit" ("um orgulho da bagunça"; Jaffe 121) äußert.

Die Ambivalenz des Verhältnisses zur deutschen Sprache wird im Kapitel "Raiva" unter anderem durch den Bezug zu Paul Celans Gedicht "Todesfuge", das in lyrischer Sprache die Judenvernichtung thematisiert, deutlich: so handelt es sich einerseits um die Sprache der Täter, der Unterdrücker, die ihre Befehle und Anweisungen im Konzentrationslager auf Deutsch geben und unmittelbar mit dem Trauma Lili Jaffes in Verbindung stehen, und ebenso um die Sprache der Unterdrückten, der deutschsprachigen Opfer, die sich - wie beispielsweise der Schriftsteller Paul Celan - nach ihrer Freilassung der deutschen Sprache als poetisches Ausdrucksmittel bedienen. Die portugiesische Übersetzung des deutschsprachigen Gedichts "Todesfuge", stößt im Werk Reflexionen über die deutsche Sprache als "Língua do dizível", als Sprache der literarischen Repräsentation der Shoah, an, ein Thema, für das Celan in der Nachkriegszeit kritisiert wurde. Die Gegenüberstellung der deutschen Sprache als "Língua da ordem" und "Língua do dizível" legt sowohl die Komplexität des geschichtlichen Phänomens der Shoah als auch die Ambiguität des Verhältnisses der Erzählinstanz zum deutschen Sprach- und Kulturraum dar und bringt nicht zuletzt die Frage nach den unterschiedlichen Funktionen von Sprache im Allgemeinen auf:

E, assim mesmo, no alemão, Paul Celan e Bruno Schulz escreveram à desordem. Foi na língua do dizível, em seu estado mais agudo, que Celan conseguiu dizer o indizível [...] (Jaffe 119).

Na língua da ordem também e possível cavar a desordem, sob os auspícios da própria língua. As mesmas palavras que serviram para esvaziar o mundo, de tão perfeitas que

Cad. Trad., Florianópolis, v. 41, no 3 p. 132-155, set-dez, 2021. 
eram suas combinações de som e sentido, de tão servis ao propósito a que eram destinadas, quando repetidas em palavra poética (nós bebemos, nós bebemos, nós bebemos; ele brada: a morte foi um dos mestres da Alemanha) ficam com seu sentido exponencialmente aguçado, até se transformarem em suportes do demônio, em fala absurda, a própria voz do inaudível. Celan desorganiza a língua ao máximo pelo próprio fato de ela ser o ápice da organização. (Jaffe 119-120)

Die literarische Transzendenz von O que os cegos estão sonhando ermöglicht nicht nur eine lebendige Auseinandersetzung mit den Erinnerungen der Mutter als Teil des Familiengedächtnisses, sondern vereint zahlreiche Stimmen unmittelbar Betroffener, die bereits im kollektiven Erfahrungsgedächtnis an die Shoah verankert sind, ohne sich den Erinnerungsdiskurs Lili Jaffes anzueignen. Gerade auch die Übersetzung ins Deutsche, bzw. die (teilweise) Aneignung der deutschen Sprache dient hier als Brücke zur Hervorholung von vergessenen, verdrängten, fragmenthaft vorhandenen Bildern und zeigt einmal mehr die Macht der Erinnerung auf.

\title{
Literarisches Übersetzen und Erinnern: Transkulturelles Shoah-Gedächtnis zwischen Europa und Brasilien
}

\begin{abstract}
Wir brauchen die Historiker, die uns diese Vergangenheit rekonstruieren, und wir brauchen die Künstler, die sie uns re-konkretisieren. Vor allem bedarf es einer Rückbindung an konkrete Lebensgeschichten und authentische Orte, um diese Erinnerung vor Verallgemeinerung, Delegation an Spezialisten und fortschreitender Entwirklichung zu schützen. (Assmann 249)
\end{abstract}


Das Buch $O$ que os cegos estão sonhando zeigt exemplarisch auf, mit welchen literarischen Darstellungsverfahren Literatur Erinnerungen rekonstruiert, ohne dabei die Shoah als Grenzereignis zu "ikonisieren" oder zu stereotypisieren. Durch trans- bzw. hypertextuelle Beziehungen wird ein „multidirektionales” (qtd in Rothberg 3) Gedächtnis konstruiert, das genügend Raum für die Vielstimmigkeit und Pluralität des Erfahrungsgedächtnisses der Shoah lässt. Die Lektüre literarischer Werke ShoahÜberlebender bzw. deren Nachkommen in der Diaspora ermöglicht eine "Rückbindung [der Shoah-Erinnerung] an konkrete Lebensgeschichten und authentische Orte", was nicht zuletzt einer "Verallgemeinerung" und "Entwirklichung" der Shoah entgegengewirkt (Assmann 249).

Die Übersetzung von $O$ que os cegos estão sonhando ins Englische bzw. ins Deutsche ${ }^{9}$ lässt im Sinne des Transcultural Turns ein Erinnern über nationale - und europäische - Grenzen hinweg zu und trägt so zur Konstruktion eines transkulturellen Shoah-Gedächtnisses jenseits der bisherigen Vorstellung national gedachter "Container-Erinnerungskulturen" (Erll, Kollektives Gedächtnis und Erinnerungskulturen. Eine Einführung, 123) bei. ${ }^{10}$

Die letzte Stufe des Übersetzens im Erinnerungsprozess, der ein Überführen der Erinnerungen Lili Jaffes und der post-memorialen Reflexionen der Tochter in ein trans-kulturelles Shoah-Gedächtnis ermöglicht, bildet die linguistische Transposition bzw. (literarische) Übersetzung des portugiesischen Textes. Als zentrales Werk der brasilianischen Gegenwartsliteratur legt $O$ que os cegos estão sonhando einen Zugang zur Vielschichtigkeit der literarischen

\footnotetext{
${ }^{9}$ Die literarische Übersetzung von $O$ que os cegos estão sonhando in Englische von Julia Sanches (What are the wild men dreaming?) erschien im Jahr 2016. Bis dato gibt es noch keine deutsche Übersetzung des Werkes.

${ }^{10}$ Siehe hierzu auch: Levy und Sznaider (Erinnerung im Globalen Zeitalter: der Holocaust, 2001), Michael Rothberg (Multidirectional Memory, 2009), Wolfgang Welsch (Transculturality - the changing form of cultures, 1999) und Astrid Erll (Travelling Memory, 2011).
} 
Inszenierung von Erinnerung und Gedächtnisarbeit und gibt Einblicke in transkulturelle, literarische Erinnerungsprozesse.

Das Konzept des Erinnerns als mehrstufiger Prozess des Übersetzens, der u.a. auf der Transposition und Transformation von Hypo- und Hypertexten basiert, legt neuartige Bedeutungskonstruktionen in einer transgenerationellen, "diachronen" Perspektive frei. Europa, insbesondere der deutsche Sprachraum, profitieren ungemein von der literarischen Übersetzung und Lektüre der brasilianischen Shoah-Literatur, denn "[d]ie ganze Wahrheit der Geschichte ist und bleibt unzugänglich, deshalb zwingt sie uns aber gerade, immer wieder neue Zugänge zu suchen" (Assmann 238).

Genau das sind die Möglichkeiten, die eine literarische Transposition von $O$ que os cegos estão sonhando in die deutsche Sprache bieten: neue Bedeutungsentfaltungen, neuartige Zugänge, aber auch Bewahrung von Bildern und Erinnerungen sowie Revision und Revitalisierung von Vergangenem als wahrhafte, andauernde, perpetuierende und dynamische Gedächtnisarbeit.

\section{Bibliografie}

Adorno, Theodor W. Prismen. Kulturkritik und Gesellschaft. München: Deutscher Taschenbuch Verlag, 1963.

Assmann, Aleida. Der lange Schatten der Vergangenheit. Erinnerungskultur und Geschichtspolitik. München: C.H. Beck, 2006.

Assmann, Aleida. Einführung in die Kulturwissenschaft. Grundbegriffe, Themen, Fragestellungen. Berlin: Erich Schmidt, 2017. 
Daniel Levy; Sznaider, Natan: Erinnerung im Globalen Zeitalter: der Holocaust. Frankfurt am Main: Suhrkamp, 2001.

Erll, Astrid. Kollektives Gedächtnis und Erinnerungskulturen. Eine Einführung. Stuttgart: Metzler ${ }^{3}, 2017$.

Erll, Astrid. "Travelling Memory". Parallax, 17/4, (2011): 4-18.

Friedländer, Saul. Memory, History, and the Extermination of the Jews of Europe. Bloomington: Indiana UP, 1993.

Gagnebin, Jeanne-Marie. Lembrar escrever esquecer. São Paulo: Editora 34, 2006.

Genette, Gérard. Palimpsests. Literature in the second Degree. Lincoln: University of Nebraska Press, 1997.

Hirsch, Marianne. Generation of Postmemory. Writing and visual culture after the Holocaust. New York: Columbia Press, 2012.

Jaffe, Noemi: O que os cegos estão sonhando. São Paulo: Editora 34, 2012.

Lachmann, Renate. Gedächtnis und Literatur. Intertextualität in der russischen Moderne. Frankfurt am Main: Suhrkamp, 1990.

Lanzmann, Claude: Shoah. Berlin: absolut Medien 2007.

Laub, Michel: Diário da Queda. São Paulo: Companhia de Letras, 2011.

Leirner, Giselda: Nas águas do mesmo rio. São Paulo: Ateliê Editorial, 2005.

Nestrovski, Arthur; Seligmann-Silva, Márcio (Hrsg.): Catástrofe e representação. São Paulo: Escuta, 2000.

Pessoa, Fernando: Fausto, tragédia subjectiva. Lisboa: Relógio d’Água, 2013. 
Rothberg, Michael. Multidirectional Memory. Remembering the Holocaust in the Age of Decolonization. Palo Alto: Stanford University Press, 2009.

Seligmann-Silva, Márcio: A história como trauma. In: Nestrovski, Arthur und Márcio Seligmann-Silva (Hrsg.): Catástrofe e representação. São Paulo: Escuta, 2000.

Seligmann-Silva, Márcio: Literatura da Shoah no Brasil. In: Revista Digital de Estudos Judaicos da UFMG 1(1) 2007, S.123-135.

Sontag, Susan. Das Leiden anderer betrachten. München/Wien: Carl Hanser, 2003.

Vaitsman, Heliete: O cisne e o aviador. Rio de Janeiro: Rocco, 2014.

Valente, Luize: Sonata em Auschwitz. Rio de Janeiro: Record, 2017.

Welsch, Wolfgang. "Transculturality - the Puzzling Form of Cultures Today". Spaces of Culture: City, Nation, World, Mike Featherstone and Scott Lash (Eds.). London: Sage, 1999. Verfügbar auf: http://www.westreadseast.info/PDF/ Readings/Welsch_Transculturality.pdf.

Recebido em: 15/04/2021

Aceito em: 20/08/2021

Publicado em setembro de 2021

Kathrin Sartingen. E-mail: kathrin.sartingen@univie.ac.at. ORCID: https:// orcid.org/0000-0001-5627-4965.

Tatjana Wais. E-mail: tatjana.wais@univie.ac.at. ORCID: https://orcid. org/0000-0002-9903-3368. 